\title{
Combining cognitive modeling and EEG to predict user behavior in a search task
}

\author{
Felix Putze (felix.putze@kit.edu), Johannes Meyer, Joscha Borné, Tanja Schultz \\ Karlsruhe Institute of Technology (KIT), Germany \\ Daniel V. Holt (daniel.holt@psychologie.uni-heidelberg.de), Joachim Funke \\ University of Heidelberg, Germany
}

Keywords: user modeling; HCI; EEG; spectral analysis; search task; cognitive modeling; temporal difference learning

In recent years, the development of intuitive humancomputer interfaces has drawn a growing interest from different research communities. One major aspect of designing such interfaces is to measure or predict the cognitive state and cognitive processes of the user. This ability would enable systems to optimize information presentation for the available cognitive resources, current goals, and cognitive context of the user and give support in critical or difficult situations.

Cognitive models have in the past successfully been applied to explain and predict user behavior and build interactive systems that respond adaptively to user state (e.g., Peebles \& Cox, 2006; Ritter, Anderson, Koedinger, \& Corbett, 2007). More recently, cognitive models have been used to predict neural activity in fMRI studies or to infer cognitive states in combination with fMRI data (e.g., Anderson, Albert \& Fincham, 2005; Anderson, Betts, Ferris \& Fincham, 2010). This approach is particularly interesting from an HCI perspective, as neural data may serve as indicators of cognitive processes that have no easily observable behavioral correlates (e.g., decision making or memory retrieval). In the present study we bring together these strands of research by combing cognitive models with neural data derived from EEG recordings to predict user behavior. To evaluate this approach, we used a paradigm based on a memory-guided search task previously employed by $\mathrm{Fu}$ and Anderson (2006). We chose this type of task as it resembles a basic type of human-computer interaction, has a structure suitable for an EEG study, and has successfully been modeled by applying a form of temporal difference learning (cf. Sutton \& Barto, 1998) implemented in the ACT-R cognitive architecture.

The focus of our analysis was (1) to explore which electrophysiological measures are suitable indicators for various aspects of cognitive user state in this task and (2) to investigate how the behavioral predictions of the cognitive model of the task could be enhanced by integrating the neural data.

\section{Method}

The task was adapted from the maze search task used by $\mathrm{Fu} \&$ Anderson (2006), which is a multi-step decision and learning paradigm. In this task, participants have to find a goal node in a hierarchical tree structure. For purposes of this study, the tree structure was presented as a file system in which a particular file had to be located. Participants start each trial at the root node of the tree. At each node, they select one of two options leading to the next node until they reach a leaf node. When the leaf node contains the target, the next trial starts. When reaching a dead end the participant is reset to the node with the last correct decision. The task consists in discovering and consistently applying the correct route from the root node to the target. To allow the manipulation of associative learning requirements, each node is labeled with a word randomly selected from a set of concrete nouns unique to the node (representation set). The correct decision option for each node is tied to this label and has to be learned. The easy version of the task used a representation set of two concrete nouns per node, the difficult version a set of four. Decision options were abstractly labeled "alpha", "beta", "gamma" and "delta" in order to eliminate spatial or semantic cues.

The task was displayed on a computer screen, showing the folder in the center and the two decision options to the left and right below the folder at each stage of the task. Responses were given by pressing one of four buttons. After instruction and practice each participant completed the task for two easy search trees for 60 trials and two difficult search trees for 120 trials. During task performance EEG was recorded from 32 channels using actiCAP active electrodes and an actiCHamp amplifier (BrainProducts, Germany). In a completed pilot study, we obtained data from 18 participants (age 19 to 26, five female).

\section{Data Analysis}

Detailed data analysis is currently in progress and will be reported in full at the conference presentation. Here, we present a short summary of the cognitive model, selected preliminary results for the behavioral data, and one potential electrophysiological indicator of learning performance. 


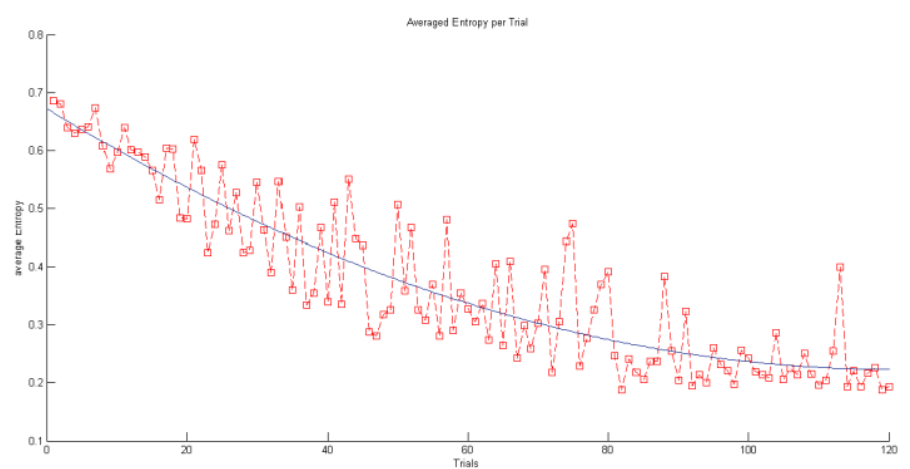

Figure 1: Development of entropy of decision probabilities for one participant in a difficult search tree; displayed per trial and as running average.

Following $\mathrm{Fu} \&$ Anderson (2006), we constructed a computational model based on temporal difference learning (TD) to simulate individual learning progress and predict the certainty of an upcoming decision. To model individual learning progress, the TD model is trained with the history of participants' decisions for each trial block in order to predict the next decision. We were able to reproduce the basic pattern of results reported in Anderson and $\mathrm{Fu}$ : The model correctly predicts faster convergence for nodes closer to the leaves of the tree as reward is only received there and propagated backwards. Additionally, we used a Softmax function to derive decision probabilities from the learned scores and calculate the entropy of the resulting distribution to derive decision confidence (Figure 1). This information can be used to identify trials traversing states for which learning has not converged yet, even if no actual error occurred.

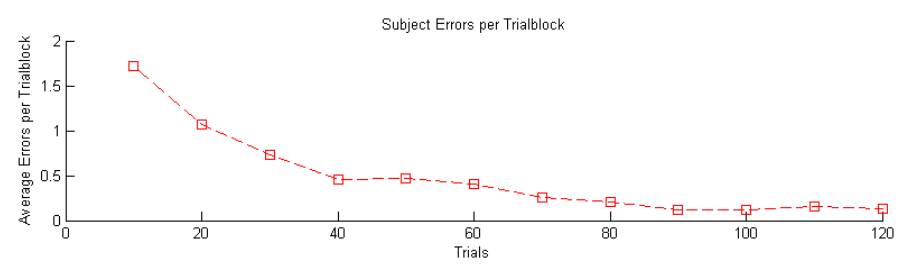

Figure 2: Error per trial block averaged over all participants for difficult search trees.

We expected to observe correlates of a number of different cognitive processes in the EEG signal. For example, we assumed that the power of oscillations in the theta band increases during memory activity (Onton, Delorme \& Makeig, 2005; Klimesch, 1998). A qualitative comparison of the aggregated behavioral data and averaged theta power in a frontal region shows that this may indeed be the case: In Figure 2, the average number of errors per trial for difficult search trees is displayed. As expected, this value drops sharply during early trials, rises again between trials 40 and 60 as interference between learned items increases, before finally converging towards a lower limit. The intermediate increase in the number of errors seems to be mirrored by a corresponding rise in theta power for those trials, as shown in Figure 3.

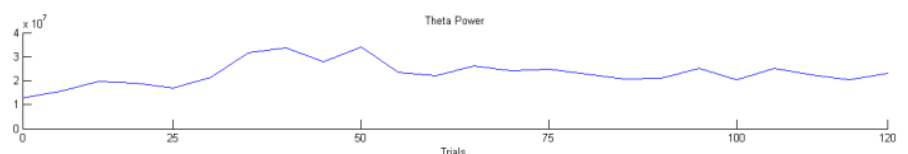

Figure 3: Spectral power in the theta band at F3 averaged over recordings for difficult search trees of three participants.

\section{Conclusion and Future Work}

The preliminary results suggest that the basic computational model is adequate and that there may be valid electrophysiological correlates of learning progress, which is a promising basis for the planned detailed analyses. Future work will concentrate on a formal integration of computational and EEG-based prediction in a Bayesian framework and the addition of further electrophysiological markers, e.g., related to decision making or error feedback. Additionally, the paradigm offers several possibilities for extension by further varying its cognitive demands (e.g., the size of the search tree or the representation set) or its semantic framing (e.g., as a web search or spatial navigation task).

\section{References}

Anderson, J., Albert, M. \& Fincham, J. (2005). Tracing Problem Solving in Real Time: fMRI Analysis of the Subject-paced Tower of Hanoi. Journal of Cognitive Neuroscience, 17, 1261-1274.

Anderson, J. R., Betts, S., Ferris, J. L., \& Fincham, J. M. (2010). Neural imaging to track mental states while using an intelligent tutoring system. PNAS, 107, 7018-7023.

Fu, W. \& Anderson, J. (2006). From Recurrent Choice to Skill Learning: A Reinforcement-Learning Model. Journal of Experimental Psychology: General, 135, 2, 183-206.

Klimesch, W. (1998). EEG alpha and theta oscillations reflect cognitive and memory performance: a review and analysis. Brain Research Reviews, 29, 169-195.

Onton, J., Delorme, A. \& Makeig, S. (2005). Frontal midline EEG dynamics during working memory. NeuroImage, 27, 341-356.

Peebles, D. J. \& Cox, A. L. (2006). Modelling Interactive Behaviour with a Rational Cognitive Architecture. In P. Zaphiris \& S. Kurniawan (Eds.). Human Computer Interaction Research in Web Design and Evaluation, London: Idea Group.

Ritter, S., Anderson, J. R., Koedinger, K. R., \& Corbett, A. (2007). Cognitive tutor: Applied research in mathematics education. Psychonomic Bulletin \& Review, 14, 249-255.

Sutton, R. S., \& Barto, A. G. (1998). Reinforcement learning: An introduction. Cambridge, MA: MIT Press. 